VOL. $61(2000)$ [353-370]

\title{
POISSON TRANSFORMS AND MIXED AUTOMORPHIC FORMS ON SEMISIMPLE LIE GROUPS
}

\author{
Min Ho Lee and Hyo Chul Myung
}

\begin{abstract}
We discuss Poisson transforms which carry sections of certain vector bundles to mixed automorphic forms, and identify vector bundles whose sections are liftings of holomorphic forms on families of Abelian varieties via Poisson transforms.
\end{abstract}

\section{INTRODUCTION}

Holomorphic mixed automorphic forms on semisimple Lie groups are automorphic forms on Hermitian symmetric domains associated to equivariant holomorphic maps of symmetric domains, and certain types of mixed automorphic forms arise as holomorphic forms on families of Abelian varieties parametrised by a locally symmetric space. The goal of this paper is to discuss Poisson transforms which carry sections of certain vector bundles to mixed automorphic forms, and identify vector bundles whose sections are liftings, via such Poisson transforms, of holomorphic forms on families of Abelian varieties.

Let $T=\left\{e^{i t} \mid-\pi \leqslant t<\pi\right\}$ be the unit circle in $\mathbb{C}$, and let $L^{1}(T)$ be the space of complex-valued integrable functions on $T$. Given an element $f \in L^{1}(T)$, if we write $\tilde{f}(t)=f\left(e^{i t}\right)$ for $t \in T$, the classical Poisson integral $\mathcal{P} f$ of $f$ is given by

$$
\mathcal{P} f\left(r e^{i \theta}\right)=\frac{1}{2 \pi} \int_{-\pi}^{\pi} \frac{1-r^{2}}{1-2 r \cos (\theta-t)+r^{2}} \tilde{f}(t) d t
$$

for $0 \leqslant r<1$ and $\theta \in \mathbb{R}$. It is well-known that $\mathcal{P} f\left(r e^{i \theta}\right)$ is a harmonic function for any $f \in L^{1}(T)$ (see, for example, [15]). If we use the normalised measure $d \tau$ for $T$, then the formula (1.1) can be written in the form

$$
(\mathcal{P} f)(z)=\int_{T} \frac{1-|z|^{2}}{|z-\tau|^{2}} f(\tau) d \tau
$$

for all $z \in U$, where $U$ is the open unit disk $\{z \in \mathbb{C}|| z \mid<1\}$. Note that $T$ and $U$ can be identified with the quotients of $S U(1,1)$ by a maximal compact subgroup and a minimal parabolic subgroup, respectively. This observation suggests a possibility of extending the notion of the Poisson integral to the case of more general semisimple Lie groups.

Received 23rd June, 1999

The second author was supported by KIAS Grant M98004.

Copyright Clearance Centre, Inc. Serial-fee code: 0004-9727/00 \$A2.00+0.00. 
Let $G$ be a semisimple Lie group of Hermitian type, and let $D=G / K$ be the Hermitian symmetric domain determined by a maximal compact subgroup $K \subset G$. Let $\sigma$ be a finite-dimensional representation of $K$ in a complex vector space, and let $P \subset G$ be a minimal parabolic subgroup. We denote by $g$ and $k$ the Lie algebras of $G$ and $K$, respectively, and let $\mathfrak{p}$ be the orthogonal complement of $\mathfrak{k}$ in $\mathfrak{g}$ relative to the Killing form. If $a_{\mathbb{C}}$ is the complexification of a maximal Abelian subspace $\mathfrak{a}$ of $\mathfrak{p}$ and if $\lambda \in \mathfrak{a}_{\mathbb{C}}^{*}$, then $\sigma$ can be extended to a representation $\sigma_{P, \lambda}$ of $P$. Thus we can consider the homogeneous vector bundle $\mathcal{W}\left(\sigma_{P, \lambda}\right)$ (respectively, $\mathcal{V}(\sigma)$ ) over $G / P$ (respectively, $G / K$ ) associated to $\sigma_{P, \lambda}$ (respectively, $\sigma$ ). In this setting, the analogue of the classical Poisson integral is the Poisson transform $\mathcal{P}_{\lambda, P}$ which assigns to each section $\phi$ of $\mathcal{W}\left(\sigma_{P, \lambda}\right)$ a section of $\mathcal{V}(\sigma)$ (see [3, Section II.3.4], [12]).

Let $G^{\prime}$ be another semisimple Lie group of Hermitian type, and let $D^{\prime}=G^{\prime} / K^{\prime}$ be the Hermitian symmetric domain associated to a maximal compact subgroup $K^{\prime} \subset$ $G^{\prime}$. Then a homomorphism $\mu: G \rightarrow G^{\prime}$ of Lie groups with $\mu(K) \subset K^{\prime}$ induces an equivariant holomorphic map $\tau: D \rightarrow D^{\prime}$. Let $\Gamma$ and $\Gamma^{\prime}$ be discrete subgroups of $G$ and $G^{\prime}$, respectively, with $\mu(\Gamma) \subset \Gamma^{\prime}$. Given automorphy factors $j: \Gamma \times D \rightarrow G L(W)$ and $j^{\prime}: \Gamma^{\prime} \times D^{\prime} \rightarrow G L\left(W^{\prime}\right)$ for finite-dimensional complex vector space $W$ and $W^{\prime}$, a holomorphic mixed automorphic form on $G$ is a $\left(W \otimes W^{\prime}\right)$-valued function on $D$ defined using the automorphy factor

$$
(\gamma, z) \mapsto j(\gamma, z) \otimes j^{\prime}(\mu(\gamma), \tau(z))
$$

(see Section 3). When $G^{\prime}$ is a symplectic group, mixed automorphic forms associated to certain automorphy factors can be identified with holomorphic forms of the highest degree on some families of Abelian varieties parametrised by a locally symmetric space.

Let $\mathfrak{a}^{\prime}$ be a subspace of $\mathfrak{g}^{\prime}=$ Lie $G$ defined as in the case of $\mathfrak{a} \subset \mathfrak{g}$. In this paper, we introduce the Poisson transform $\mathcal{P}_{\lambda, \lambda^{\prime}}$ associated to $\lambda \in \mathfrak{a}_{\mathbb{C}}^{*}$ and $\lambda^{\prime} \in \mathfrak{a}_{\mathbb{C}}^{\prime *}$ defined on the space of sections of a homogeneous vector bundle $\mathcal{W}\left(\sigma_{\lambda, \lambda^{\prime}}\right)$ over $G / P$. Given a torsion-free discrete subgroup $\Gamma$ of $G$ and a $\Gamma$-invariant section $\phi$ of $\mathcal{W}\left(\sigma_{\lambda, \lambda^{\prime}}\right)$, we prove that $\mathcal{P}_{\lambda, \lambda^{\prime}} \phi$ is a holomorphic mixed automorphic form. Using the correspondence between mixed automorphic forms and holomorphic forms on families of Abelian varieties, we identify vector bundles whose sections are Poisson transform liftings of holomorphic forms of the highest degree on some families of Abelian varieties.

\section{Canonical automorphy factors}

In this section we describe holomorphic automorphic forms on semisimple Lie groups of Hermitian type and discuss canonical automorphy factors for such Lie groups.

Let $G$ be a connected semisimple Lie group, and let $K$ be a maximal compact subgroup of $G$. We assume that $G$ is of Hermitian type, which means that the associated symmetric space $D=G / K$ has a $G$-invariant complex structure. Thus $D$ is a Hermitian 
symmetric space and can be realised as a bounded domain in $\mathbb{C}^{k}$ for some positive integer $k$. Let $W$ be a finite-dimensional complex vector space, and let $S$ be a subgroup of $G$. A map $\mathcal{J}: S \times D \rightarrow G L(W)$ is called an automorphy factor of $S$ if it satisfies the following conditions:

(i) For fixed $g \in S$, the map $z \mapsto \mathcal{J}(g, z), D \rightarrow G L(W)$ is holomorphic.

(ii) For all $g_{1}, g_{2} \in S$ and $z \in D$, we have

$$
\mathcal{J}\left(g_{1} g_{2}, z\right)=\mathcal{J}\left(g_{1}, g_{2} z\right) \cdot \mathcal{J}\left(g_{2}, z\right) .
$$

Let $\Gamma$ be a torsion-free discrete subgroup of $G$. Then the complex structure on $D$ induces the structure of a complex manifold on the locally symmetric space $X=\Gamma \backslash D$, and we can define automorphic forms on $D$ as follows (see [2]).

Definition 2.1: Let $\mathcal{J}: \Gamma \times D \rightarrow G L(W)$ be an automorphy factor of $\Gamma$. A holomorphic automorphic form on $D$ (or on $G$ ) of type $\mathcal{J}$ for $\Gamma$ is a holomorphic map $f: D \rightarrow W$ that satisfies

$$
f(\gamma z)=\mathcal{J}(\gamma, z) \cdot f(z)
$$

for all $z \in D$ and $\gamma \in \Gamma$.

Given an automorphy factor $\mathcal{J}: \Gamma \times D \rightarrow G L(W)$, we can construct the associated vector bundle $\mathcal{M}(\Gamma, \mathcal{J})$ on the locally symmetric space $X=\Gamma \backslash D$ as follows. Let the discrete subgroup $\Gamma$ of $G$ act on $D \times W$ by

$$
\gamma \cdot(z, w)=(\gamma z, \mathcal{J}(\gamma, z) w)
$$

for all $\gamma \in \Gamma$ and $(z, w) \in D \times W$. The fact that this operation indeed defines an action of $\Gamma$ on $D \times W$ follows from the condition (2.1). We set

$$
\mathcal{M}(\Gamma, \mathcal{J})=\Gamma \backslash D \times W
$$

where the quotient is taken with respect to the above action of $\Gamma$ on $D \times W$. Then the natural projection $D \times W \rightarrow D$ induces the structure of a holomorphic vector bundle on the induced map

$$
\varpi: \mathcal{M}(\Gamma, \mathcal{J}) \rightarrow X=\Gamma \backslash D
$$

with fibre $W$. Let $\Gamma_{0}(X, \mathcal{M}(\Gamma, \mathcal{J}))$ denote the space of holomorphic sections of $\mathcal{M}(\Gamma, \mathcal{J})$ over $X$, that is, holomorphic maps $s: X \rightarrow \mathcal{M}(\Gamma, \mathcal{J})$ such that $\varpi \circ s=1_{X}$. Given a section $s: X \rightarrow \mathcal{M}(\Gamma, \mathcal{J})$ of $\mathcal{M}(\Gamma, \mathcal{J})$ and an element $z \in D$ we have

$$
s(\Gamma z)=\left[\left(z, w_{z}\right)\right] \in \mathcal{M}(\Gamma, \mathcal{J})=\Gamma \backslash D \times W
$$

for some $w_{z} \in W$, where $\Gamma z \in X$ and $\left[\left(z, w_{z}\right)\right] \in \mathcal{M}(\Gamma, \mathcal{J})$ denote the elements corresponding to $z \in D$ and $\left(z, w_{z}\right) \in D \times W$, respectively. We define $f_{s}: D \rightarrow W$ by 
$f_{s}(z)=w_{z}$ for all $z \in D$. Then it can be shown that the map $s \mapsto f_{s}$ determines a canonical isomorphism between $\Gamma_{0}(X, \mathcal{M}(\Gamma, \mathcal{J}))$ and the space of holomorphic automorphic forms on $D$ of type $\mathcal{J}$ for $\Gamma$.

We shall now describe the construction of the canonical automorphy factor of the semisimple Lie group of $G$ of Hermitian type. Let $I$ be a $G$-invariant complex structure on $D=G / K$. Then for each $z \in D$ it determines a complex structure $I_{z}$ on the tangent space $T_{z}(D)$. Let $\mathfrak{g}$ and $\mathfrak{k}$ be the Lie algebras of $G$ and $K$, respectively, and let $\mathfrak{g}=\mathfrak{k}+\mathfrak{p}$ be the corresponding Cartan decomposition of $\mathfrak{g}$. If $z_{0}$ is the point in $D$ with $K z_{0}=z_{0}$, then we can identify $\mathfrak{p}$ with the tangent space $T_{z_{0}}(D)$; hence we obtain a complex structure $I_{z_{0}}$ on $\mathfrak{p}$. We set

$$
\mathfrak{p}_{ \pm}=\left\{X \in \mathfrak{p}_{\mathbb{C}} \mid I_{z_{0}}(X)= \pm i X\right\}
$$

and denote by $P_{+}, P_{-}$the $\mathbb{C}$-subgroups of $G_{\mathbb{C}}$ corresponding to $\mathfrak{p}_{+}, \mathfrak{p}_{-}$, respectively; here $(\cdot)_{\mathbb{C}}$ denotes the complexification. Then we have

$$
P_{+} \cap K_{\mathbb{C}} P_{-}=\{1\}, \quad G \subset P_{+} K_{\mathbb{C}} P_{-}, \quad G \cap K_{\mathbb{C}} P_{-}=K
$$

(see [16, Lemma II.4.2], [14]). If $g \in P_{+} K_{\mathbb{C}} P_{-} \subset G_{\mathbf{C}}$, we denote by $(g)_{+} \in P_{+},(g)_{0} \in K_{\mathbb{C}}$ and $(g)_{-} \in P_{-}$the components of $g$ such that

$$
g=(g)_{+} \cdot(g)_{0} \cdot(g)_{-} .
$$

Let $\left(G_{\mathbb{C}} \times \mathfrak{p}_{+}\right)_{*}$ denote the subset of $G_{\mathbb{C}} \times \mathfrak{p}_{+}$consisting of elements $(g, z)$ such that $g \cdot \exp z \in P_{+} K_{\mathbb{C}} P_{-}$, and set

$$
J(g, z)=(g \cdot \exp z)_{0} \in K_{\mathbb{C}}
$$

for $(g, z) \in\left(G_{\mathbb{C}} \times \mathbf{p}_{+}\right)_{*}$. If we identify the Hermitian symmetric domain $D$ with a subset of $\mathfrak{p}_{+}$using the Harish-Chandra embedding $D \hookrightarrow \mathfrak{p}_{+}$(see [16, Section II.4]), then we have

$$
G \times D \subset\left(G_{\mathbf{C}} \times \mathfrak{p}_{+}\right)_{*}
$$

Thus we obtain a map $J: G \times D \rightarrow K_{\mathbf{C}}$ which satisfies the condition

$$
J\left(g_{1} g_{2}, z\right)=J\left(g_{1}, g_{2} z\right) \cdot J\left(g_{2}, z\right)
$$

for $g_{1}, g_{2} \in G$ and $z \in D$. The map $J$ is called the canonical automorphy factor of $G$. Let $\sigma: K \rightarrow G L(W)$ be a representation of $K$ in a complex vector space $W$, and extend it to a representation of $K_{\mathbb{C}}$. Thus, using (2.4), we see that $\sigma \circ J: G \times D \rightarrow G L(W)$ is an automorphy factor in the sense of Definition 2.1 . 


\section{MIXED AUTOMORPHIC FORMS}

Mixed automorphic forms were introduced by Hunt and Meyer [4] who showed that holomorphic two-forms on an elliptic surface can be identified with certain mixed automorphic forms. Such mixed automorphic forms are defined by using automorphy factors that involve the monodromy representation and the period map of the given elliptic surface. On the other hand, holomorphic forms on the fibre product of elliptic surfaces correspond to mixed automorphic forms of higher weights (see, for example [7]). Mixed automorphic forms of several variables and their connection with holomorphic forms on families of Abelian varieties have also been studied recently (see $[8,10,9,11]$ ). In this section we describe holomorphic mixed automorphic forms on semisimple Lie groups.

Let $G, K, D=G / K, \Gamma$ and $X=\Gamma \backslash D$ be as in Section 2. Let $G^{\prime}$ be another semisimple Lie group of Hermitian type, and let $D^{\prime}=G^{\prime} / K^{\prime}$ be the Hermitian symmetric domain associated to a maximal compact subgroup $K^{\prime}$ of $G^{\prime}$. We assume that there are a homomorphism $\mu: G \rightarrow G^{\prime}$ of Lie groups and a holomorphic map $\tau: D \rightarrow D^{\prime}$ that are equivariant, that is, they satisfy $\tau(g z)=\mu(g) \tau(z)$ for all $g \in G$ and $z \in D$. In particular, we have $\mu(K) \subset K^{\prime}$. Let $\Gamma^{\prime}$ be a torsion-free discrete subgroup of $G^{\prime}$ such that $\mu(\Gamma) \subset \Gamma^{\prime}$. Then the complex structure on $D^{\prime}$ induces the structure of a complex manifold on the locally symmetric space $X^{\prime}=\Gamma^{\prime} \backslash D^{\prime}$, and the holomorphic map $\tau$ induces a map $X \rightarrow X^{\prime}$.

DEFINITION 3.1: Let $j: \Gamma \times D \rightarrow G L(W)$ and $j^{\prime}: \Gamma^{\prime} \times D^{\prime} \rightarrow G L\left(W^{\prime}\right)$ be automorphy factors of $\Gamma$ and $\Gamma^{\prime}$, respectively, where $W$ and $W^{\prime}$ are finite-dimensional complex vector spaces. A holomorphic mixed automorphic form on $D$ (or on $G$ ) of type $\left(j, j^{\prime}, \mu, \tau\right)$ for $\Gamma$ is a holomorphic map $f: D \rightarrow W \otimes W^{\prime}$ that satisfies

$$
f(\gamma z)=j(\gamma, z) \otimes j^{\prime}(\mu(\gamma), \tau(z)) \cdot f(z)
$$

for all $z \in D$ and $\gamma \in \Gamma$.

Let $j$ and $j^{\prime}$ be as in Definition 3.1, and let $\Gamma$ act on $D \times\left(W \otimes W^{\prime}\right)$ by

$$
\gamma \cdot(z, w)=\left(\gamma z, j(\gamma, z) \otimes j^{\prime}(\mu(\gamma), \tau(z)) w\right)
$$

for all $\gamma \in \Gamma, z \in D$ and $w \in W \otimes W^{\prime}$. We denote by $\mathcal{M}\left(\Gamma, j, j^{\prime}\right)$ the associated quotient space, that is,

$$
\mathcal{M}\left(\Gamma, j, j^{\prime}\right)=\Gamma \backslash D \times\left(W \otimes W^{\prime}\right) .
$$

Then the natural projection $D \times\left(W \otimes W^{\prime}\right) \rightarrow D$ determines the structure of a holomorphic vector bundle on the induced map

$$
\varpi: \mathcal{M}\left(\Gamma, j, j^{\prime}\right) \rightarrow X=\Gamma \backslash D .
$$

with fibre $W \otimes W^{\prime}$. We denote by $\Gamma_{0}\left(X, \mathcal{M}\left(\Gamma, j, j^{\prime}\right)\right)$ the space of holomorphic sections of $\mathcal{M}\left(\Gamma, j, j^{\prime}\right)$ over $X$, that is, holomorphic maps $s: X \rightarrow \mathcal{M}\left(\Gamma, j, j^{\prime}\right)$ satisfying $\varpi \circ s=1_{X}$. 
LEMMA 3.2. The space $\Gamma_{0}\left(X, \mathcal{M}\left(\Gamma, j, j^{\prime}\right)\right)$ of sections of $\mathcal{M}\left(\Gamma, j, j^{\prime}\right)$ is isomorphic to the space of holomorphic mixed automorphic forms on $D$ of type $\left(j, j^{\prime}, \mu, \tau\right)$ for $\Gamma$.

Proof: A holomorphic mixed automorphic form on $D$ of type $\left(j, j^{\prime}, \mu, \tau\right)$ for $\Gamma$ is a holomorphic automorphic form with respect to the automorphy factor

$$
(g, z) \mapsto j(g, z) \otimes j^{\prime}(\mu(g), \tau(z))
$$

of $\Gamma$. On the other hand, we see that $\mathcal{M}\left(\Gamma, j, j^{\prime}\right)$ is simply the vector bundle $\mathcal{M}(\Gamma, \mathcal{J})$ considered in (2.2) for $\mathcal{J}$ equal to this automorphy factor. Hence the lemma follows from the usual isomorphism between $\mathcal{M}(\Gamma, \mathcal{J})$ and the space of automorphic forms of type $\mathcal{J}$ described in Section 2.

\section{HOMOGENEOUS VECTOR BUNDLES}

Let $\mathfrak{G}$ be a Lie group, and let $\mathfrak{H}$ be a closed subgroup of $\mathfrak{G}$. Let $\mathfrak{G} / \mathfrak{H}$ denote the set $\{g \mathfrak{H} \mid g \in \mathfrak{G}\}$ of left cosets modulo $\mathfrak{H}$, and let $p: \mathfrak{G} \rightarrow \mathfrak{G} / \mathfrak{H}$ be the natural projection $p(g)=g \mathfrak{H}$. Then $\mathfrak{G} / \mathfrak{H}$ has a unique manifold structure such that $p$ is smooth and for each $g \mathfrak{H} \in \mathfrak{G} / \mathfrak{H}$ there is a neighbourhood $U$ of $g \mathfrak{H}$ and a smooth map $\mu: U \rightarrow \mathfrak{G}$ such that $p \circ \mu=\mathrm{id}_{U}$. The quotient space $\mathfrak{G} / \mathfrak{H}$ with such a manifold structure is called a homogeneous manifold.

DEFinition 4.1: Let $M=\mathfrak{G} / \mathfrak{H}$ be a homogeneous manifold. A vector bundle $E$ over $M$ is called a homogeneous vector bundle if $\mathfrak{G}$ acts on $E$ on the left and the $\mathfrak{G}$-action satisfies the following conditions:

(i) If $E_{x}$ denotes the fibre of $E$ over $x \in M$, then $g \cdot E_{x}=E_{g x}$ for all $x \in M$ and $g \in \mathfrak{G}$.

(ii) The map $E_{x} \rightarrow E_{g x}$ induced by each $g \in \mathfrak{G}$ is linear for all $x \in M$.

We shall now construct a homogeneous vector bundle associated to a representation of $\mathfrak{H}$. Let $M=\mathfrak{G} / \mathfrak{H}$ be a homogeneous manifold, and let $\eta$ be a representation of $\mathfrak{H}$ in a finite-dimensional complex vector space $V$. Then $\mathfrak{H}$ acts on the product $\mathfrak{G} \times V$ on the right by

$$
(g, v) \cdot h=\left(g h, \eta(h)^{-1} v\right)
$$

for all $h \in \mathfrak{H}$ and $(g, v) \in \mathfrak{G} \times V$. We set

$$
\mathcal{V}(\eta)=\mathfrak{G} \times V / \mathfrak{H}
$$

where the quotient is taken with respect to the action of $\mathfrak{H}$ on $\mathfrak{G} \times V$ given by (4.1). The natural projection $\mathfrak{G} \times V \rightarrow \mathfrak{G}$ induces the map $\pi: \mathcal{V}(\eta) \rightarrow M$ which has the structure of a vector bundle with fibre $V$ (see [17]). It can be shown that $\mathcal{V}(\eta)$ is a homogeneous vector bundle over $M$. Let $\Gamma_{0}(M, \mathcal{V}(\eta))$ be the space of sections of $\mathcal{V}(\eta)$, that is, smooth 
maps $s: M \rightarrow \mathcal{V}(\eta)$ such that $\pi \circ s=1_{M}$. If $s: M \rightarrow \mathcal{V}(\eta)$ is a section of $\mathcal{V}(\eta)$ and $g \in G$, we have

$$
s(g H)=\left[\left(g, v_{g}\right)\right] \in \mathcal{V}(\eta)
$$

for some $v_{g} \in V$, where $\left[\left(g, v_{g}\right)\right]$ denotes the element of $\mathcal{V}(\eta)$ corresponding to $\left(g, v_{g}\right) \in$ $D \times V$. We set $f_{s}(g)=v_{g}$ for all $g \in G$. Then for each $h \in H$ we have

$$
s(g H)=s(g h H)=\left[\left(g h, v_{g h}\right)\right]=\left[\left(g h, v_{g h}\right) \cdot h^{-1}\right]=\left[\left(g, \eta(h) v_{g h}\right)\right] .
$$

Thus we see that

$$
f_{s}(g)=\eta(h) v_{g h}=\eta(h) f_{s}(g h) .
$$

Therefore each section $s \in \Gamma_{0}(M, \mathcal{V}(\eta))$ of $\mathcal{V}(\eta)$ can be identified with a smooth function $f: \mathfrak{G} \rightarrow V$ on $\mathfrak{G}$ satisfying

$$
f(g h)=\eta(h)^{-1} f(g)
$$

for all $(g, v) \in G \times V$ and $h \in H$.

Suppose now that $\mathfrak{G}$ is a semisimple Lie group of Hermitian type, and let $\mathfrak{K}$ be a maximal compact subgroup of $\mathfrak{G}$ so that the associated homogeneous manifold $M=\mathfrak{G} / \mathfrak{K}$ becomes a Hermitian symmetric domain. Let $\eta$ be a representation of $\mathfrak{K}$ in $V$, and let $\Delta$ be a torsion-free discrete subgroup of $\mathfrak{G}$. We denote by $\Gamma_{0}(\Delta, \mathcal{V}(\eta))^{\Delta}$ the space of $\Delta$ invariant sections of the associated homogeneous vector bundle $\mathcal{V}(\eta)$. Thus each element of $\Gamma_{0}(M, \mathcal{V}(\eta))^{\Delta}$ can be identified with a holomorphic function $f: G \rightarrow V$ such that

$$
f(\delta g k)=\eta(k)^{-1} f(g)
$$

for all $\delta \in \Delta, k \in \mathfrak{K}$ and $g \in \mathfrak{G}$.

LEMMA 4.2. Let $J: \mathfrak{B} \times M \rightarrow \mathfrak{K}_{\mathbb{C}}$ be the canonical automorphy factor of $\mathfrak{B}$, and let $J_{\eta}=\eta \circ J: \mathfrak{G} \times M \rightarrow G L(V)$ be the automorphy factor of $\mathfrak{G}$ associated to $\eta$. If $z_{0} \in M$ is the element with $\mathfrak{K} z_{0}=z_{0}$, then the map $[(g, v)] \mapsto\left[\left(g z_{0}, v\right)\right]$ determines a canonical isomorphism between $\Gamma_{0}(M, \mathcal{V}(\eta))^{\Delta}$ and the space of all holomorphic automorphic forms on $M$ of type $J_{\eta}$ for $\Delta$.

Proof: See, for example [13, Theorem II.4.1].

\section{POISSON TRANSFORMS}

Let $G, K, \mathfrak{g}, \mathfrak{k}$ and $\mathfrak{p}$ be as in Section 2 with $\mathfrak{g}=\mathfrak{k}+\mathfrak{p}$, and let $\mathfrak{a}$ be a maximal Abelian subspace of $\mathfrak{p}$. Then we obtain the Iwasawa decomposition

$$
\mathfrak{g}=\mathfrak{k}+\mathfrak{a}+\mathfrak{n}
$$


of $\mathfrak{g}$, where $\mathfrak{n}$ is a nilpotent subalgebra of $\mathfrak{g}$ (see for example [5, Section V.2]). Let $A$ and $N$ be subgroups of $G$ corresponding to the Lie algebras $\mathfrak{a}$ and $\mathfrak{n}$, respectively, so that we obtain the Iwasawa decomposition $G=K A N$ of $G$. Let $M$ be the centraliser of $A$ in $K$, and set $P=M A N$, which is a minimal parabolic subgroup of $G$. We shall write any element $g \in G$ in the form $g=\kappa(g) \cdot e^{H(g)} \cdot n$ with $\kappa(g) \in K, H(g) \in \mathfrak{a}$ and $n \in N$.

Let $G^{\prime}, K^{\prime}$ and $\mu: G \rightarrow G^{\prime}$ be as in Section 3. We shall denote various objects associated to $G^{\prime}$ by adding the prime symbol to the corresponding objects for $G$. Thus $G^{\prime}=K^{\prime} A^{\prime} N^{\prime}$ is the Iwasawa decomposition of $G^{\prime}$, and each element $g^{\prime} \in G^{\prime}$ can be decomposed in the form

$$
g^{\prime}=\kappa^{\prime}\left(g^{\prime}\right) \cdot e^{H^{\prime}\left(g^{\prime}\right)} \cdot n^{\prime}
$$

with $\kappa^{\prime} \in K^{\prime}, H^{\prime}\left(g^{\prime}\right) \in \mathfrak{a}^{\prime}$ and $n^{\prime} \in N^{\prime}$. Let $P^{\prime}=M^{\prime} A^{\prime} N^{\prime}$ be the associated minimal parabolic subgroup of $G^{\prime}$, where $M^{\prime}$ is the centraliser of $A^{\prime}$ in $K^{\prime}$. We assume that $\mu(A) \subset A^{\prime}$ and $\mu(N) \subset N^{\prime}$.

Let $\sigma$ (respectively, $\sigma^{\prime}$ ) be an irreducible representation of $K$ (respectively, $K^{\prime}$ ) in a finite-dimensional complex vector space $W$ (respectively, $W^{\prime}$ ), so that $\sigma \otimes\left(\sigma^{\prime} \circ \mu\right)$ becomes a representation of $K$ in $W \otimes W^{\prime}$. Given an element $\lambda \in \mathfrak{a}_{\mathbb{C}}^{*}$ we define the representation $\sigma_{\lambda, P}$ of $P$ in $W$ by

$$
\sigma_{\lambda, P}(\operatorname{man})=e^{(-\lambda+\rho) H(a)} \sigma(m),
$$

for all $m \in M, a \in A$ and $n \in N$, where $\rho$ is the half-sum of $\operatorname{dim}\left(\mathfrak{g}_{\alpha}\right) \alpha$ over the positive roots $\alpha$ of $(\mathfrak{g}, \mathfrak{a})$. Similarly, if $\lambda^{\prime} \in \mathfrak{a}_{\mathbb{C}}^{\prime *}$ and if $\rho^{\prime}$ is the half-sum of $\operatorname{dim}\left(\mathfrak{g}_{\alpha^{\prime}}^{\prime}\right) \alpha^{\prime}$ over the positive roots $\alpha^{\prime}$ of $\left(\mathfrak{g}^{\prime}, \mathfrak{a}^{\prime}\right)$, we denote by $\sigma_{\lambda^{\prime}, P^{\prime}}^{\prime}$ the representation of $P^{\prime}$ in $W^{\prime}$ defined by

$$
\sigma_{\lambda^{\prime}, P^{\prime}}^{\prime}\left(m^{\prime} a^{\prime} n^{\prime}\right)=e^{\left(-\lambda^{\prime}+\rho^{\prime}\right) H^{\prime}\left(a^{\prime}\right)} \sigma^{\prime}\left(m^{\prime}\right),
$$

for all $m^{\prime} \in M^{\prime}, a^{\prime} \in A^{\prime}$ and $n^{\prime} \in N^{\prime}$. We then define the representation $\sigma_{\lambda, \lambda^{\prime}}$ of $P$ in $W \otimes W^{\prime}$ by

$$
\sigma_{\lambda, \lambda^{\prime}}=\sigma_{\lambda, P} \otimes\left(\sigma_{\lambda^{\prime}, P^{\prime}}^{\prime} \circ \mu\right)
$$

so that we have

$$
\sigma_{\lambda, \lambda^{\prime}}(\operatorname{man})=e^{-(\lambda-\rho) H(a)-\left(\lambda^{\prime}-\rho^{\prime}\right) H^{\prime}(\mu(a))}\left(\sigma \otimes\left(\sigma^{\prime} \circ \mu\right)\right)(m)
$$

for $m \in M, a \in A$ and $n \in N$.

Let $\mathcal{V}\left(\sigma \otimes\left(\sigma^{\prime} \circ \mu\right)\right)$ (respectively, $\left.\mathcal{W}\left(\sigma_{\lambda, \lambda^{\prime}}\right)\right)$ be the homogeneous vector bundle on $G / K$ (respectively, $G / P)$ associated to the representation $\sigma \otimes\left(\sigma^{\prime} \circ \mu\right)$ (respectively, $\left.\sigma_{\lambda, \lambda^{\prime}}\right)$. Using (4.2) and (5.1), we see that a section $\phi \in \Gamma_{0}\left(G / P, \mathcal{W}\left(\sigma_{\lambda, \lambda^{\prime}}\right)\right)$ of the bundle $\mathcal{W}\left(\sigma_{\lambda, \lambda^{\prime}}\right)$ can be regarded as a smooth function $\phi: G \rightarrow W \otimes W^{\prime}$ satisfying

$$
\begin{aligned}
\phi(g m a n) & =\sigma_{\lambda, \lambda^{\prime}}(\operatorname{man})^{-1} \phi(g) \\
& =e^{(\lambda-\rho) H(a)+\left(\lambda^{\prime}-\rho^{\prime}\right) H^{\prime}(\mu(a))}\left(\sigma \otimes\left(\sigma^{\prime} \circ \mu\right)\right)(m)^{-1} \phi(g)
\end{aligned}
$$


for $g \in G, m \in M$ and $a \in A$. Similarly, a section $\psi \in \Gamma_{0}\left(G / K, \mathcal{V}\left(\sigma \otimes\left(\sigma^{\prime} \circ \mu\right)\right)\right)$ of the vector bundle $\mathcal{V}\left(\sigma \otimes\left(\sigma^{\prime} \circ \mu\right)\right)$ can be identified with a function $\psi: G \rightarrow W \otimes W^{\prime}$ such that

$$
\psi(g k)=\left(\sigma \otimes\left(\sigma^{\prime} \circ \mu\right)\right)(k)^{-1} \psi(g)
$$

for all $k \in K$ and $g \in G$.

DEFinition 5.1: Let $\phi$ be an element of $\Gamma_{0}\left(G / P, \mathcal{W}\left(\sigma_{\lambda, \lambda^{\prime}}\right)\right)$, that is, a smooth function $\phi: G \rightarrow W \otimes W^{\prime}$ satisfying (5.2). The Poisson transform $\mathcal{P}_{\lambda, \lambda^{\prime}} \phi$ of $\phi$ is a $\left(W \otimes W^{\prime}\right)$-valued function on $G$ given by

$$
\left(\mathcal{P}_{\lambda, \lambda^{\prime}} \phi\right)(g)=\int_{K}\left(\sigma \otimes\left(\sigma^{\prime} \circ \mu\right)\right)(k) \phi(g k) d k
$$

for all $g \in G$.

LEMMA 5.2. Let $f$ be a continuous function on $K$ that is right invariant under $K \cap M$. Then we have

$$
\int_{K} f(k) d k=\int_{K} e^{-2 \rho H\left(g^{-1} k\right)} f\left(\kappa\left(g^{-1} k\right)\right) d k
$$

for $g \in G$.

Proof: See for example [5, p. 170].

Lemma 5.3. The Poisson transform $\mathcal{P}_{\lambda, \lambda^{\prime}} \phi$ of an element $\phi \in \Gamma_{0}\left(G / P, \mathcal{W}\left(\sigma_{\lambda, \lambda^{\prime}}\right)\right)$ can be written in the form

$$
\left(\mathcal{P}_{\lambda, \lambda^{\prime}} \phi\right)(g)=\int_{K} e^{-(\lambda+\rho) H\left(g^{-1} k\right)-\left(\lambda^{\prime}-\rho^{\prime}\right) H^{\prime}\left(\mu\left(g^{-1} k\right)\right)}\left(\sigma \otimes\left(\sigma^{\prime} \circ \mu\right)\right)\left(\kappa\left(g^{-1} k\right)\right) \phi(k) d k
$$

for all $g \in G$.

Proof: If $m \in K \cap M$, then by (5.2) we have

$$
\phi(g k m)=\left(\sigma \otimes\left(\sigma^{\prime} \circ \mu\right)\right)(m)^{-1} \phi(g k)
$$

for $g \in G$ and $k \in K$. Thus the function

$$
k \mapsto\left(\sigma \otimes\left(\sigma^{\prime} \circ \mu\right)\right)(k) \phi(g k)
$$

is right invariant under $K \cap M$, and hence, applying Lemma 5.2 to this function, we obtain

$$
\begin{aligned}
\left(\mathcal{P}_{\lambda, \lambda^{\prime}} \phi\right)(g) & =\int_{K}\left(\sigma \otimes\left(\sigma^{\prime} \circ \mu\right)\right)(k) \phi(g k) d k \\
& =\int_{K} e^{-2 \rho H\left(g^{-1} k\right)}\left(\sigma \otimes\left(\sigma^{\prime} \circ \mu\right)\right)\left(\kappa\left(g^{-1} k\right)\right) \phi\left(g \kappa\left(g^{-1} k\right)\right) d k
\end{aligned}
$$


for $g \in G$. Let $g^{-1} k=\kappa\left(g^{-1} k\right) \cdot a_{1} \cdot n_{1}$ with $a_{1} \in A$ and $n_{1} \in N$. Then we have

$$
g \kappa\left(g^{-1} k\right)=k n_{1}^{-1} a_{1}^{-1}=k a_{1}^{-1} n^{\prime}
$$

for some $n^{\prime} \in N$ because $A$ normalizes $N$. Hence, using (5.2), we see that

$$
\begin{aligned}
\phi\left(g \kappa\left(g^{-1} k\right)\right) & =\phi\left(k a_{1}^{-1} n^{\prime}\right) \\
& =e^{(\lambda-\rho) H\left(a_{1}^{-1}\right)+\left(\lambda^{\prime}-\rho^{\prime}\right) H^{\prime}\left(\mu\left(a_{1}^{-1}\right)\right)} \phi(k) \\
& =e^{-(\lambda-\rho) H\left(a_{1}\right)-\left(\lambda^{\prime}-\rho^{\prime}\right) H^{\prime}\left(\mu\left(a_{1}\right)\right)} \phi(k) \\
& =e^{-(\lambda-\rho) H\left(g^{-1} k\right)-\left(\lambda^{\prime}-\rho^{\prime}\right) H^{\prime}\left(\mu\left(g^{-1} k\right)\right)} \phi(k) .
\end{aligned}
$$

Thus, for $g \in G$ and $k \in K$, we have

$$
\begin{aligned}
&\left(\mathcal{P}_{\lambda, \lambda^{\prime}} \phi\right)(g)=\int_{K} e^{-2 \rho H\left(g^{-1} k\right)}\left(\sigma \otimes\left(\sigma^{\prime} \circ \mu\right)\right)\left(\kappa\left(g^{-1} k\right)\right) \\
& \times e^{-(\lambda-\rho) H\left(g^{-1} k\right)-\left(\lambda^{\prime}-\rho^{\prime}\right) H^{\prime}\left(\mu\left(g^{-1} k\right)\right)} \phi(k) d k
\end{aligned}
$$

and hence the lemma follows.

Let $\Gamma$ be a torsion-free discrete subgroup of $G$ as in Section 3 , and consider the left-action of $\Gamma$ on $G \times\left(W \otimes W^{\prime}\right)$ defined by

$$
\gamma \cdot(g, w)=(\gamma g, w)
$$

for $\gamma \in \Gamma, g \in G$ and $w \in W \otimes W^{\prime}$. Since this action commutes with the one given by (4.1) for $\eta=\sigma \otimes\left(\sigma^{\prime} \circ \mu\right)$ and $V=W \otimes W^{\prime}$ that was used for the construction of a homogeneous vector bundle, the homogeneous vector bundle

$$
\mathcal{V}\left(\sigma \otimes\left(\sigma^{\prime} \circ \mu\right)\right) \rightarrow D=G / K
$$

associated to $\sigma \otimes\left(\sigma^{\prime} \circ \mu\right)$ induces the vector bundle

$$
\mathcal{V}\left(\Gamma, \sigma \otimes\left(\sigma^{\prime} \circ \mu\right)\right)=\Gamma \backslash \mathcal{V}\left(\sigma \otimes\left(\sigma^{\prime} \circ \mu\right)\right)
$$

over the locally symmetric space $X=\Gamma \backslash D=\Gamma \backslash G / K$ with fibre $W \otimes W^{\prime}$. Similarly, we obtain the vector bundle

$$
\mathcal{W}\left(\Gamma, \sigma_{\lambda, \lambda^{\prime}}\right)=\Gamma \backslash \mathcal{W}\left(\sigma_{\lambda, \lambda^{\prime}}\right)
$$

over the space $\Gamma \backslash G / P$ whose fibre is again $W \otimes W^{\prime}$. Thus each section

$$
\phi \in \Gamma_{0}\left(\Gamma \backslash G / P, \mathcal{W}\left(\Gamma, \sigma_{\lambda, \lambda^{\prime}}\right)\right)
$$

of $\mathcal{W}\left(\Gamma, \sigma_{\lambda, \lambda^{\prime}}\right)$ is a $\Gamma$-invariant section of the homogeneous vector bundle $\mathcal{W}\left(\sigma_{\lambda, \lambda^{\prime}}\right)$ over $G / P$, and it can be identified with a smooth function $\phi: G \rightarrow W \otimes W^{\prime}$ on $G$ satisfying (5.2) and

$$
\phi(\gamma g)=\phi(g)
$$


for all $\gamma \in \Gamma$ and $g \in G$. Similarly, a section $\psi \in \Gamma_{0}\left(X, \mathcal{V}\left(\Gamma, \sigma \otimes\left(\sigma^{\prime} \circ \mu\right)\right)\right.$ of $\nu\left(\Gamma, \sigma \otimes\left(\sigma^{\prime} \circ \mu\right)\right)$ can be regarded as a smooth function $\psi: G \rightarrow W \otimes W^{\prime}$ satisfying (5.3) and $\psi(\gamma g)=\psi(g)$ for $\gamma \in \Gamma$ and $g \in G$.

Now we state our main theorem in this section, which implies that each section of the vector bundle $\mathcal{W}\left(\Gamma, \sigma_{\lambda, \lambda^{\prime}}\right)$ over $\Gamma \backslash G / P$ can be regarded as a lifting, via the Poisson transform map $\mathcal{P}_{\lambda, \lambda^{\prime}}$, of a holomorphic mixed automorphic form on $D$ for $\Gamma$.

THEOREM 5.4. Let $\phi$ be an element of $\Gamma_{0}\left(\Gamma \backslash G / P, \mathcal{W}\left(\Gamma, \sigma_{\lambda, \lambda^{\prime}}\right)\right)$ regarded as a $\left(W \otimes W^{\prime}\right)$-valued smooth function on $G$ satisfying (5.2) and (5.7), and let $J_{\sigma}=\sigma \circ J$ and $J_{\sigma^{\prime}}^{\prime}=\sigma^{\prime} \circ J^{\prime}$, where $J: G \times D \rightarrow K_{\mathbb{C}}$ and $J^{\prime}: G^{\prime} \times D^{\prime} \rightarrow K_{\mathbf{C}}^{\prime}$ are the canonical automorphy factors of $G$ and $G^{\prime}$, respectively, described in Section 2. Then the Poisson transform $\mathcal{P}_{\lambda, \lambda^{\prime}} \phi$ of $\phi$ is a holomorphic mixed automorphic form on $D$ of type $\left(J_{\sigma}, J_{\sigma^{\prime}}^{\prime}, \mu, \tau\right)$ for $\Gamma$.

Proof: Let $\phi$ be an element of $\Gamma_{0}\left(\Gamma \backslash G / P, \mathcal{W}\left(\Gamma, \sigma_{\lambda, \lambda^{\prime}}\right)\right)$. Using Lemma 5.3, for each $g \in G$ and $k_{1} \in K$ we obtain

$$
\begin{aligned}
\left(\mathcal{P}_{\lambda, \lambda^{\prime}} \phi\right)\left(g k_{1}\right)= & \int_{K} e^{-(\lambda+\rho) H l\left(\left(g k_{1}\right)^{-1} k-\left(\lambda^{\prime}-\rho^{\prime}\right)\left(H^{\prime} \mu\left(\left(g k_{1}\right)^{-1} k\right)\right)\right.} \\
& \times\left(\sigma \otimes\left(\sigma^{\prime} \circ \mu\right)\right)\left(\kappa\left(\left(g k_{1}\right)^{-1} k\right)\right) \phi(k) d k \\
= & \int_{K} e^{-(\lambda+\rho) H\left(k_{1}^{-1} g^{-1} k\right)-\left(\lambda^{\prime}-\rho^{\prime}\right) H^{\prime}\left(\mu\left(k_{1}\right)^{-1} \mu\left(g^{-1} k\right)\right)} \\
& \times\left(\sigma \otimes\left(\sigma^{\prime} \circ \mu\right)\right)\left(\kappa\left(k_{1}^{-1} g^{-1} k\right)\right) \phi(k) d k \\
= & \int_{K} e^{-(\lambda+\rho) H\left(g^{-1} k\right)-\left(\lambda^{\prime}-\rho^{\prime}\right) H^{\prime}\left(\mu\left(g^{-1} k\right)\right)} \\
& \times\left(\sigma \otimes\left(\sigma^{\prime} \circ \mu\right)\right)\left(k_{1}^{-1} \kappa\left(g^{-1} k\right)\right) \phi(k) d k \\
= & \left(\sigma \otimes\left(\sigma^{\prime} \circ \mu\right)\right)\left(k_{1}^{-1}\right) \cdot \int_{K} e^{-(\lambda+\rho) H\left(g^{-1} k\right)-\left(\lambda^{\prime}-\rho^{\prime}\right) H^{\prime}\left(\mu\left(g^{-1} k\right)\right)} \\
& \times\left(\sigma \otimes\left(\sigma^{\prime} \circ \mu\right)\right)\left(\kappa\left(g^{-1} k\right)\right) \phi(k) d k \\
= & \left(\sigma \otimes\left(\sigma^{\prime} \circ \mu\right)\right)\left(k_{1}\right)^{-1} \cdot\left(\mathcal{P}_{\lambda, \lambda^{\prime}} \phi\right)(g),
\end{aligned}
$$

which implies that $\mathcal{P}_{\lambda, \lambda^{\prime}} \phi$ is a smooth section of $\mathcal{V}\left(\sigma \otimes\left(\sigma^{\prime} \circ \mu\right)\right)$. Since the function

$$
g \mapsto e^{-(\lambda+\rho) H\left(g^{-1}\right)-\left(\lambda^{\prime}-\rho^{\prime}\right) H^{\prime}\left(\mu\left(g^{-1}\right)\right)}\left(\sigma \otimes\left(\sigma^{\prime} \circ \mu\right)\right)\left(\kappa\left(g^{-1}\right)\right)
$$

is analytic and $K$ is compact, using (5.5), we see that $\mathcal{P}_{\lambda, \lambda^{\prime}} \phi$ is also analytic. Furthermore, since $D=G / K$ is assumed to have a $G$-invariant complex structure, it follows that $\mathcal{P}_{\lambda, \lambda^{\prime}} \phi$ is in fact a holomorphic section of the bundle $\mathcal{V}\left(\sigma \otimes\left(\sigma^{\prime} \circ \mu\right)\right)$. On the other hand, using (5.4), we obtain

$$
\left(\mathcal{P}_{\lambda, \lambda^{\prime}} \phi\right)(\gamma g)=\int_{K}\left(\sigma \otimes\left(\sigma^{\prime} \circ \mu\right)\right)(k) \phi(\gamma g k) d k=\left(\mathcal{P}_{\lambda, \lambda^{\prime}} \phi\right)(g)
$$

for all $\gamma \in \Gamma$ and $g \in G$. Therefore $\mathcal{P}_{\lambda, \lambda^{\prime}} \phi$ is $\Gamma$-invariant, and hence it follows that $\mathcal{P}_{\lambda, \lambda^{\prime}} \phi$ is an element of $\Gamma_{0}\left(X, \mathcal{V}\left(\Gamma, \sigma \otimes\left(\sigma^{\prime} \circ \mu\right)\right)\right)$. However, using Lemma 4.2, we see that 
$\Gamma_{0}\left(X, \mathcal{V}\left(\Gamma, \sigma \otimes\left(\sigma^{\prime} \circ \mu\right)\right)\right)$ is isomorphic to the space of holomorphic automorphic forms on $D$ of type $\left(\sigma \otimes\left(\sigma^{\prime} \circ \mu\right)\right) \circ J$ for $\Gamma$, where $J: G \times D \rightarrow K_{\mathbf{C}}$ is the canonical automorphy factor of $G$. Now using (2.3), we obtain

$$
\begin{aligned}
\left(\left(\sigma \otimes\left(\sigma^{\prime} \circ \mu\right)\right) \circ J\right)(g, z) & =\left(\sigma \otimes\left(\sigma^{\prime} \circ \mu\right)\right)\left((g \cdot \exp z)_{0}\right) \\
& =\sigma\left((g \cdot \exp z)_{0}\right) \otimes \sigma^{\prime}\left(\mu(g \cdot \exp z)_{0}\right) \\
& =\sigma\left((g \cdot \exp z)_{0}\right) \otimes \sigma^{\prime}\left(\mu(g \cdot \exp \tau(z))_{0}\right) \\
& =(\sigma \circ J)(g, z) \otimes\left(\sigma^{\prime} \circ J^{\prime}\right)(\nu(g), \tau(z)) \\
& =J_{\sigma}\left((g \cdot \exp z)_{0}\right) \otimes J_{\sigma^{\prime}}^{\prime}(\mu(g), \tau(z)) .
\end{aligned}
$$

for all $(g, z) \in G \times D$. Hence it follows that $\mathcal{P}_{\lambda, P} \phi$ is a holomorphic mixed automorphic form on $D$ of type $\left(J_{\sigma}, J_{\sigma^{\prime}}^{\prime}, \mu, \tau\right)$ for $\Gamma$.

\section{KUGA FIBRE VARIETIES}

In this section we describe families of Abelian varieties, known as Kuga fibre varieties, parametrised by a locally symmetric space and discuss a correspondence between holomorphic forms of the highest degree on Kuga fibre varieties and certain mixed automorphic forms.

Let $V$ be a real vector space of dimension $2 n$ defined over $\mathbb{Q}$, and let $\beta$ be a nondegenerate alternating bilinear form on $V$ also defined over $\mathbb{Q}$. We denote by $S p(V, \beta)$ the symplectic group of the form $\beta$ on $V$. Now we consider the homomorphism $\mu: G \rightarrow G^{\prime}$ described in Section 3 for $G^{\prime}=\mathbb{G}^{\prime}(\mathbb{Q})$ with $\mathbb{G}^{\prime}=S p(V, \beta)$, and set

$$
D=G / K, \quad \mathcal{H}=G^{\prime} / K^{\prime},
$$

where $K$ and $K^{\prime}$ are maximal compact subgroups of $G$ and $G^{\prime}$, respectively, as before. Let $\Gamma \subset \mathbb{G}(\mathbb{Q})$ be a torsion-free arithmetic subgroup. Then the locally symmetric space $X=\Gamma \backslash D$ has a natural structure of a complex quasi-projective variety (see [1]). Since it is assumed that $\mu(K) \subset K^{\prime}$, the homomorphism $\mu$ induces an equivariant holomorphic map $\tau: D \rightarrow \mathcal{H}$ satisfying

$$
\tau(g z)=\mu(g) \tau(z)
$$

for all $g \in G$ and $z \in D$. Let $G \ltimes_{\mu} V$ be the semidirect product of $G$ and $V$ with respect to the action of $G$ on $V$ via $\mu$. Thus $G \times_{\mu} V$ consists of the elements $(g, v)$ in $G \times V$, and its multiplication operation is given by

$$
(g, v) \cdot\left(g^{\prime}, v^{\prime}\right)=\left(g g^{\prime}, \mu(g) v^{\prime}+v\right)
$$

for $g, g^{\prime} \in G$ and $v, v^{\prime} \in V$. The space $G \ltimes_{\mu} V$ acts on $D \times V$ by

$$
(g, v) \cdot\left(z, v^{\prime}\right)=\left(g z, \mu(g) v^{\prime}+v\right)
$$


for all $g \in G, z \in D$ and $v, v^{\prime} \in V$. Let $L$ be a lattice in $V$ such that $\mu(\Gamma) L \subset L$ and $\beta(L, L) \subset \mathbb{Z}$. Regarding $\mathcal{H}$ as the set of complex structures on $V$, each element $z \in D$ determines a complex vector space $\left(V, I_{\tau(z)}\right)$, where $I_{\tau(z)}$ is the complex structure on $V$ corresponding to $\tau(z) \in \mathcal{H}$. Let $z_{0}$ be a fixed element of $D$, and let $I_{0}$ be the complex structure on $V$ corresponding to the element $\tau\left(z_{0}\right)$ of $\mathcal{H}$. Let $V_{\mathbf{C}}=V \otimes_{\mathbf{R}} \mathbb{C}$ be the complexification of $V$, and denote by $V_{+}$and $V_{-}$the subspaces of $V_{\mathbb{C}}$ defined by

$$
V_{ \pm}=\left\{v \in V_{\mathbf{C}} \mid I_{0} v= \pm i v\right\}
$$

so that we have

$$
V_{\mathbf{C}}=V_{+} \oplus V_{-}, \quad V_{+}=\bar{V}_{-} .
$$

Then each element $v$ in $\left(V, I_{\tau(z)}\right)$ determines an element

$$
\xi(z, v)=v_{z}=v_{+}-\tau(z) v_{-}=v_{+}-I_{\tau(z)} v_{-}
$$

of the subspace $V_{+}$of $V_{\mathbb{C}}$, where the elements $v_{ \pm}$denote the $V_{ \pm}$-components of $v \in V \subset$ $V_{\mathrm{C}}=V_{+} \oplus V_{-}:$We set

$$
W=\coprod_{z \in D}\left(V, I_{\tau(z)}\right)
$$

the disjoint union of the vector spaces $\left(V, I_{\tau(z)}\right)$ with complex structure $I_{\tau(z)}$ for the elements $z \in D$. Then the map

$$
W \rightarrow D \times V_{+}, \quad(z, v) \mapsto(z, \xi(z, v))
$$

determines a bijection $W \cong D \times V_{+}$and a $\mathbb{C}$-linear isomorphism $\left(V, I_{\tau(z)}\right) \cong\{z\} \times V_{+}$. Thus the natural projection map $W \rightarrow D$ has the structure of a holomorphic vector bundle with fibre $V_{+}$. Now $G \ltimes_{\mu} V$ acts on $W$ by

$$
(g, v) \cdot(z, w)=(g z, \mu(g)(w+v))
$$

for $(g, v) \in G \times_{\mu} V$ and $(z, w) \in W$, that is, $z \in D$ and $w \in\left(V, I_{\tau(z)}\right)$. Using the isomorphism between $W$ and $D \times V_{+}$given in (6.3), we see that, if $w=\xi(z, u)$ with $u \in\left(V, I_{\tau(z)}\right), G \times_{\mu} V$ acts on $D \times V_{+}$by

$$
\begin{aligned}
(g, v) \cdot(z, w) & =(g z, \xi(g z, \mu(g) u)+\xi(g z, \mu(g) v)) \\
& =\left(g z, \xi(g z, \mu(g) u)+(\mu(g) v)_{g z}\right)
\end{aligned}
$$

for $(g, v) \in G \times_{\mu} V$ and $(z, w) \in D \times V_{+}$.

We extend the alternating bilinear form $\beta$ on $V$ to $\beta: V_{\mathbb{C}} \times V_{\mathbb{C}} \rightarrow \mathbb{C}$, and let $\left\{u_{1}, \ldots, u_{2 n}\right\}$ a symplectic basis of $V_{\mathbf{C}}$ for $i \beta$ such that $\left\{u_{1}, \ldots, u_{n}\right\}$ is a basis of $V_{+}$and 


$$
\begin{aligned}
i \beta\left(u_{j}, u_{k}\right) & =0=i \beta\left(u_{n+j}, u_{n+k}\right), \\
i \beta\left(u_{j}, u_{n+j}\right) & =0=i \beta\left(u_{n+k}, u_{k}\right)
\end{aligned}
$$

for $1 \leqslant j, k \leqslant n$.

Lemma 6.1. Let $(g, v) \in G \alpha_{\mu} V$ be such that

$$
\mu(g)=\left(\begin{array}{ll}
A_{\mu} & B_{\mu} \\
C_{\mu} & D_{\mu}
\end{array}\right) \in S p(V, \beta)
$$

with respect to the basis $\left\{u_{1}, \ldots, u_{2 n}\right\}$ described above. Then the action in (6.5) can be written in the form

$$
(g, v) \cdot(z, w)=\left(g z,{ }^{t}\left(C_{\mu} \tau(z)+D_{\mu}\right)^{-1} w+(\mu(g) v)_{g z}\right)
$$

for all $(z, w) \in D \times V_{+}$.

Proof: By (7.13) in [16, Chapter IV] we have

$$
\xi(g z, \mu(g) u)={ }^{t}\left(C_{\mu} \tau(z)+D_{\mu}\right)^{-1} \cdot \xi(z, u)
$$

for $g \in G$ and $(z, u) \in W=\coprod_{z \in D}\left(V, I_{\tau(z)}\right)$. Thus the lemma follows from this and (6.5).

Then the action of $G \times_{\mu} V$ on $W$ in (6.4) induces an action of $\Gamma \times_{\mu} V$ on $W$. We set

$$
Y=\Gamma \ltimes_{\mu} L \backslash W .
$$

The natural projection map $W \rightarrow D$ determines the structure of a fibre bundle on the induced projection map $\pi: Y \rightarrow X=\Gamma \backslash D$ whose fibre over $\Gamma z \in X$ with $z \in D$ is the quotient $\left(V, I_{\tau(z)}\right) / L$ of the complex vector space $\left(V, I_{\tau(z)}\right)$ by the lattice $L$. The complex torus $\left(V, I_{\tau(z)}\right) / L$ is in fact an Abelian variety because the alternating bilinear form $\beta$ can be used as a Riemann form. Thus we obtain a fibre bundle $\pi: Y \rightarrow X$ whose fibres are Abelian varieties of the form $\left(V, I_{\tau(z)}\right) / L$. The total space $Y$ of such a fibre bundle is called a Kuga fibre variety (see [6], [16, Chapter IV]).

Let $J_{H}: G \times D \rightarrow K_{\mathbf{C}}$ be the canonical automorphy factor of $G$, and let Ad : $G \rightarrow$ $G L(\mathfrak{g})$ be the adjoint representation of $G$. Thus we have $\operatorname{Ad}(g)=d \nu_{g}$ for each $g \in G$, where $\nu_{g}: G \rightarrow G$ is the homomorphism given by $\nu_{g}(h)=g h g^{-1}$ for all $h \in G$. We extend Ad to the representation Ad : $G_{\mathbf{C}} \rightarrow G L(\mathbf{g c})$ of the complexification $G_{\mathbb{C}}$ of $G$. Then we see that

$$
\operatorname{Ad}(k) \cdot \mathfrak{p}_{+} \subset \mathfrak{p}_{+}
$$


for all $k \in K_{\mathbf{C}}$. We denote by $\operatorname{Ad}_{\mathfrak{p}_{+}}: K_{\mathbf{C}} \rightarrow G L\left(\mathfrak{p}_{+}\right)$the representation of $K_{\mathbf{C}}$ in $\mathfrak{p}_{+}$given by

$$
\operatorname{Ad}_{p_{+}}(k)=\left.\operatorname{Ad}(k)\right|_{p_{+}}
$$

for $k \in K_{\mathbb{C}}$, and define the automorphy factor $j_{H}: G \times D \rightarrow \mathbb{C}^{\times}$by

$$
j_{H}(g, z)=\operatorname{det}\left[\operatorname{Ad}_{\mathfrak{p}_{+}}\left(J_{H}(g, z)\right)\right]
$$

for $(g, z) \in G \times D$. Then for each $g \in G$ the map $z \mapsto j_{H}(g, z)$ is simply the Jacobian map of the transformation $z \mapsto g z$ of $D$. We also define the automorphy factor $j_{V}$ : $G^{\prime} \times D^{\prime} \rightarrow \mathbb{C}^{\times}$by

$$
j_{V}\left(g^{\prime}, Z\right)=\operatorname{det}(C Z+D)
$$

for $Z \in \mathcal{H}$ and

$$
g^{\prime}=\left(\begin{array}{ll}
A & B \\
C & D
\end{array}\right) \in S p(n, \mathbb{R}) .
$$

Now we state a theorem that describes a connection between mixed automorphic forms and holomorphic forms on Kuga fibre varieties. This extends the result in [10, Theorem 4.2], where the case of compact $X$ was considered.

THEOREM 6.2. Let $Y^{m}$ be the fibre product of $m$ copies of the Kuga fibre variety $Y$ over $X$ constructed above, and let $\Omega^{k+m n}$ be the sheaf of holomorphic $(k+m n)$-forms on $Y^{m}$. Then the space $H^{0}\left(Y^{m}, \Omega^{k+m n}\right)$ of sections of $\Omega^{k+m n}$ is canonically isomorphic to the space of mixed automorphic forms on $D$ of type $\left(j_{H}^{-1}, j_{V}^{m}, \mu, \tau\right)$ for $\Gamma$.

Proof: Since $D$ is a Hermitian symmetric domain, it can be realised as a bounded domain in $\mathbb{C}^{k}$ with $k=\operatorname{dim}_{\mathbf{C}} D$. Let $z=\left(z_{1}, \ldots, z_{k}\right)$ be the global coordinate system for $D$. Recalling that each fibre of $Y^{m}$ is of the form $\left(V_{+} / L\right)^{m}$ with $V_{+} / L$ a complex torus, let $\zeta^{(j)}=\left(\zeta_{1}^{(j)}, \ldots, \zeta_{n}^{(j)}\right)$ be a coordinate system for the complex vector space $V_{+}$ for $1 \leqslant j \leqslant n$. Let $\Phi$ be a holomorphic $(k+m n)$-form on $Y^{m}$. Then $\Phi$ can be regarded as a holomorphic $(k+m n)$-form on $D \times V_{+}^{m}$ that is invariant under the action of $\Gamma \times L^{m}$. Thus there is a holomorphic function $f_{\Phi}(z, \zeta)$ on $D \times V_{+}^{m}$ such that

$$
\Phi(z, \zeta)=f_{\Phi}(z, \zeta) d z \wedge d \zeta^{(1)} \wedge \cdots \wedge d \zeta^{(m)},
$$

where $z=\left(z_{1}, \ldots, z_{k}\right) \in D, \zeta=\left(\zeta^{(1)}, \ldots, \zeta^{(m)}\right) \in V_{+}^{m}$ and

$$
\zeta^{(j)}=\left(\zeta_{1}^{(j)}, \ldots, \zeta_{n}^{(j)}\right) \in V_{+}, \quad d \zeta^{(j)}=d \zeta_{1}^{(j)} \wedge \cdots \wedge d \zeta_{n}^{(j)}
$$

for $1 \leqslant j \leqslant m$. Give an element $z_{0} \in D$, the restriction of the form $\Phi$ to the fibre $Y_{z_{0}}^{n}$ of $Y^{n}$ over $\Gamma z_{0} \in X=\Gamma \backslash D$ is the holomorphic $m n$-form

$$
\Phi\left(z_{0}, \zeta\right)=f_{\Phi}\left(z_{0}, \zeta\right) d z \wedge d \zeta^{(1)} \wedge \cdots \wedge d \zeta^{(m)},
$$


where $\zeta \mapsto f_{\Phi}\left(z_{0}, \zeta\right)$ is a holomorphic function on $Y_{z_{0}}^{n}$. However, $Y_{z_{0}}^{n}$ is a complex torus of dimension $m n$, and therefore is compact. Since any holomorphic function on a compact complex manifold is constant, we see that $f_{\Phi}$ is a function of $z$ only. Thus we have

$$
\Phi(z, \zeta)=\tilde{f}_{\Phi}(z) d z \wedge d \zeta^{(1)} \wedge \cdots \wedge d \zeta^{(m)},
$$

where $\tilde{f}$ is a holomorphic function on $D$. In order to use the condition that $\Phi$ is invariant under the action of $\Gamma \times L^{m}$, consider an element

$$
(\gamma, l)=\left(\gamma, l_{1}, \ldots, l_{m}\right) \in \Gamma \times L^{m} .
$$

Then its action on $\mathrm{dz}$ is given by

$$
d z \circ(\gamma, l)=j_{H}(\gamma, z) d z,
$$

since $z \mapsto j_{H}(\gamma, z)$ is the Jacobian map for the transformation $z \mapsto \gamma z$ of $D$ into itself as stated above. On the other hand, by Lemma 6.1 the action of $\Gamma \times L^{m}$ on

$$
d \zeta^{(j)}=\left(d \zeta_{1}^{(j)}, \ldots, d \zeta_{n}^{(j)}\right) \in V_{+}
$$

is given by

$$
\begin{aligned}
d \zeta^{(j)} \circ(\gamma, l) & =\bigwedge_{i=1}^{n} d\left[{ }^{t}\left(C_{\mu} \tau(z)+D_{\mu}\right)^{-1} \zeta_{i}^{(j)}+\left(\mu(\gamma) l_{j}\right)_{\gamma z}\right] \\
& =\operatorname{det}\left(C_{\mu} \tau(z)+D_{\mu}\right)^{-1} \bigwedge_{i=1}^{n} d \zeta_{i}^{(j)} \\
& =j_{V}(\mu(\gamma), \tau(z))^{-1} d \zeta^{(j)}
\end{aligned}
$$

for $1 \leqslant j \leqslant m$, where

$$
\mu(\gamma)=\left(\begin{array}{cc}
A_{\mu} & B_{\mu} \\
C_{\mu} & D_{\mu}
\end{array}\right) \in S p(V, \beta)
$$

Thus we obtain

$$
\Phi \circ(\gamma, l)=\tilde{f}_{\Phi}(\gamma z) j_{H}(\gamma, z) j_{V}(\mu(\gamma), \tau(z))^{-m} d z \wedge d \zeta^{(1)} \wedge \cdots \wedge d \zeta^{(m)}
$$

hence it follows that

$$
\tilde{f}_{\Phi}(\gamma z)=j_{H}(\gamma, z)^{-1} j_{V}(\mu(\gamma), \tau(z))^{m} \widetilde{f}_{\Phi}(z)
$$

for all $\gamma \in \Gamma$ and $z \in D$. On the other hand, given a mixed automorphic form $f$ on $D$ of type $\left(j_{H}^{-1}, j_{V}^{m}, \mu, \tau\right)$, we define the $(k+m n)$-form $\Phi_{f}$ on $Y^{m}$ by

$$
\Phi_{f}(z, \zeta)=f(z) d z \wedge d \zeta^{(1)} \wedge \cdots \wedge d \zeta^{(m)} .
$$


Then for $(\gamma, l)=\left(\gamma, l_{1}, \ldots, l_{m}\right) \in \Gamma \times L^{m}$ we have

$$
\begin{aligned}
\left(\Phi_{f} \circ(\gamma, l)\right)(z, \zeta) & =f(\gamma z) j_{H}(\gamma, z) j_{V}(\mu(\gamma), \tau(z))^{-m} d z \wedge d \zeta^{(1)} \wedge \cdots \wedge d \zeta^{(m)} \\
& =f(z) d z \wedge d \zeta^{(1)} \wedge \cdots \wedge d \zeta^{(m)}=\Phi_{f}(z, \zeta) .
\end{aligned}
$$

Thus $\Phi_{f}$ is a $\left(\Gamma \times L^{m}\right)$-invariant $(k+m n)$-form on $D \times V_{+}^{m}$, and therefore the map $f \mapsto \Phi_{f}$ gives an isomorphism between the space of mixed automorphic forms on $D$ of type $\left(j_{H}^{-1}, j_{V}^{m}, \mu, \tau\right)$ and the space $H^{0}\left(Y^{m}, \Omega^{k+m n}\right)$.

Corollary 6.3. Let $\sigma: K \rightarrow \mathbb{C}^{\times}$and $\sigma^{\prime}: K^{\prime} \rightarrow \mathbb{C}^{\times}$be the representations of $K$ and $K^{\prime}$, respectively, in the one-dimensional complex vector space $\mathbb{C}$ given by

$$
\sigma(k)=\operatorname{det}\left[A d_{\mathfrak{p}_{+}}(k)\right]^{-1}, \quad \sigma^{\prime}\left(k^{\prime}\right)=\left(\operatorname{det} k^{\prime}\right)^{m}
$$

for $k \in K$ and $k^{\prime} \in K^{\prime}$, and let $\phi$ be an element of $\Gamma_{0}\left(\Gamma \backslash G / P, \mathcal{W}\left(\Gamma, \sigma_{\lambda, \lambda^{\prime}}\right)\right)$. Then the Poisson transform $\mathcal{P}_{\lambda, \lambda^{\prime}} \phi$ of $\phi$ can be identified with a holomorphic $(k+m n)$-form on the fibre product $Y^{m}$ of $m$ copies of the Kuga fibre variety $Y$ over $X$. In other words, holomorphic sections of $\mathcal{W}\left(\Gamma, \sigma_{\lambda, \lambda^{\prime}}\right)$ are Poisson transform liftings of holomorphic forms of the highest degree on $Y^{m}$.

Proof: This follows from Theorem 6.2 and the fact that the canonical automorphy factor $J_{V}: G^{\prime} \times D^{\prime} \rightarrow K_{\mathbb{C}}^{\prime}$ of the symplectic group $G^{\prime}$ is given by

$$
J_{V}\left(g^{\prime}, Z\right)={ }^{t}(C Z+D)
$$

for $Z \in \mathcal{H}$ and $g^{\prime}=\left(\begin{array}{c}A \\ C\end{array}\right) \in S p(n, \mathbb{R})$ (see for example [16, Chapter II, Corollary 7.4]).

\section{REFERENCES}

[1] W. Baily and A. Borel, 'Compactification of arithmetic quotients of bounded symmetric domains', Ann. of Math. 84 (1966), 442-528.

[2] A. Borel, 'Introduction to automorphic forms', in Algebraic Groups and Discontinuous Subgroups, Boulder, Colo. 1965, Proc. Sympos. Pure Math. 9 (Amer. Math. Soc., Providence, R.I., 1966), pp. 199-210.

[3] S. Helgason, Geometric analysis on symmetric spaces (Amer. Math. Soc., Providence, R.I., 1994).

[4] B. Hunt and W. Meyer, 'Mixed automorphic forms and invariants of elliptic surfaces', Math. Ann. 271 (1985), 53-80.

[5] A. Knapp, Representation theory of semisimple groups: An overview based on examples (Priceton University Press, Princeton, 1986).

[6] M. Kuga, Fiber varieties over a symmetric space whose fibers are abelian varieties I, II (Univ. of Chicago, Chicago, 1963/64).

[7] M.H. Lee, 'Mixed cusp forms and holomorphic forms on elliptic varieties', Pacific J. Math. 132 (1988), 363-370. 
[8] M. H. Lee, 'Mixed Siegel modular forms and Kuga fiber varieties', Mlinois J. Math. 38 (1994), 692-700.

[9] M. H. Lee, 'Mixed automorphic forms on semisimple Lie groups', Mlinois J. Math. 40 (1996), 464-478.

[10] M. H. Lee, 'Mixed automorphic vector bundles on Shimura varieties', Pacific J. Math. 173 (1996), 105-126.

[11] M. H. Lee, 'Mixed Hilbert modular forms and families of abelian varieties', Glasgow Math. J. 39 (1997), 131-140.

[12] K. Minemura, 'Invariant differential operators and spherical sections on a homogeneous vector bundle', Tokyo J. Math. 15 (1992), 231-245.

[13] D. Mumford, 'Families of abelian varieties', in Algebraic Groups and Discontinuous Subgroups, Boulder, Colo. 1965, Proc. Sympos. Pure Math. 9 (Amer. Math. Soc., Providence, R.I., 1966), pp. 347-351.

[14] S. Murakami, Cohomology of vector-valued forms on symmetric spaces (Univ. of Chicago, Chicago, 1966).

[15] W. Rudin, Real and complex analysis, (Third edition) (McGraw-Hill, New York, 1987).

[16] I. Satake, Algebraic structures of symmetric domains (Princeton Univ. Press, Princeton, 1980).

[17] N. Wallach, Harmonic analysis in homogeneous spaces (Marcel Dekker, New York, 1973).

Department of Mathematics

University of Northern Iowa

Cedar Falls, IA 50614

United States of America

e-mail: lee@math.uni.edu
Korea Institute for Advanced Study and KAIST

207-43 Chunryangri-dong

Dongdaemoon-ku

Seoul 130-012

Korea

e-mail: hm@kias.re.kr 